\title{
Chipping machines: disc and drum energy requirements
}

\author{
Alessio Facello, ${ }^{1}$, Eugenio Cavallo, ${ }^{1}$ Raffaele Spinelli ${ }^{2}$ \\ 'CNR IMAMOTER, Torino, Italy; ${ }^{2}$ CNR IVALSA, Sesto Fiorentino (FI), Italy
}

\begin{abstract}
Air pollution and fossil fuel reserves exhaustion are increasing the importance of the biomass-derived products, in particular wood, as source of clean and renewable energy for the production of electricity or steam. In order to improve the global efficiency and the entire production chain, we have to evaluate the energetic aspects linked to the process of transformation, handling and transport of these materials. This paper reports results on a comparison between two chippers of similar size using different cutting technology: disc and drum tool respectively. During trials, fuel consumption, PT0 torque and speed, processing time and weight of processed material were recorded. Power demand, fuel consumption, specific energy and productivity were computed. The machine was fed with four different feedstock types (chestnut logs, poplar logs, poplar branches, poplar sawmill residues). 15 repetitions for each combination of feedstock-tool were carried out. The results of this study show that the disc tool requires, depending on the processed material, from 12 to $18 \%$ less fuel per unit of material processed than the drum tool, and consequently, from 12 to $16 \%$ less specific energy. In particular, the highest difference between tools was found in branches processing whereas the smallest was in poplar logs. Furthermore the results of the investigation indicate, that, in testing conditions, the productivity of drum tool is higher (8\%) than disc tool.
\end{abstract}

\section{Introduction}

The use of fossil energy sources like petrol and carbon has a strong consequence on greenhouse effect, climate change and acidification of water and soil (de Wit and Faaij, 2010). Biomass-derived products are an important source of renewable energy and can reduce the global pollution. Traditional agricultural crops, dedicated energy crops and residues from agriculture and forestry have high potential for the pro-

Correspondence: Alessio Facello, Strada delle Cacce 73, Torino, Italy.

Tel. +39.011 .3977721 .

E-mail: a.facello@ima.to.cnr.it

Key words: chipping, energy, fuel consumption, power, tractor.

(C) Copyright A. Facello et al., 2013

Licensee PAGEPress, Italy

Journal of Agricultural Engineering 2013; XLIV(s2):e75

doi:10.4081/jae.2013.s2.e75

This article is distributed under the terms of the Creative Commons Attribution Noncommercial License (by-nc 3.0) which permits any noncommercial use, distribution, and reproduction in any medium, provided the original author(s) and source are credited. duction of heat, steam, electricity and transportation fuels (Chum and Overend, 2001).

Various type of processing machines are used to facilitate handling and transport of wood (Hillring, 2006), that is one of the most important source of biomass. The energetic aspects linked to the process of transformation, handling and transport of these materials have to be considered in order to optimize and improve the entire process.

This paper refers result on a comparison between two chippers of similar size using different cutting technology: disc and drum tool respectively. Trials has been performed to investigate the differences in terms of energy requirements, hourly production and type of raw material processed by the two machines.

\section{Materials and methods}

Chippers used during tests are small-scale machines designed for operate with medium power agricultural tractor. They have the same log diameter capacity (250 mm max) and they use different cutting technologies: disc tool and drum tool respectively. In both cases chippers mount two blades with a offset of 13 and $15 \mathrm{~mm}$ respectively, in order to obtain the same final cut length. The disc machine has a piece breaker and the drum chipper mounted a $50 \times 50 \mathrm{~mm}$ mesh sieve to reduce the oversize particles output.

A $100 \mathrm{~kW}$ agricultural tractor powered the machines, setting the Power-Take-Off to the speed recommended by the chippers manufacturer: $540 \mathrm{rpm}$ for the disc chipper and $750 \mathrm{rpm}$ for the drum chipper respectively.

The tractor was equipped with transducers measuring:

Torque and rotating speed of the power-take-off;

Fuel consumption;

Fuel temperature.

A strain-gauge based torque meter, giving a rated voltage output of $2 \mathrm{mV} \cdot \mathrm{V}^{-1} @ 2250 \mathrm{Nm}$, was used to measure continuously the torque adsorbed by the machines. Power-take-off rotating speed was detected with a 60 tooth gear coupled with a magnetic pick-up that give a pulse output directly proportional to the speed $\left(1 \mathrm{~Hz} \cdot \mathrm{rpm}^{-1}\right.$, $\max 5000 \mathrm{rpm}$ ).

Fuel consumption measurement was carried out with a volumetric fuel meter directly connected with the engine feed line (send and return pipe). This device returns a pulse signal proportional to the volume of fuel that flow through the meter. Rated output is 2000 pulse per $\mathrm{dm}^{3}$, that is equivalent at $1.8 \mathrm{~Hz}$ per $\mathrm{dm}^{3} \cdot \mathrm{h}^{-1}$ in terms of hourly consumption. The fuel meter was equipped with two PT100 thermocouples connected respectively to the send and return lines in order to know the fluid temperature and deduce the fuel density.

Trials were performed processing different feedstock types (poplar logs, chestnut logs, poplar sawmill residues and poplar branches). The moisture content was in the range $44-52 \%$ and was determined with the gravimetric method according to the European standard CEN/TS 14774-2 collecting one sample at the end of each repetition. For each combination feedstock-machine were performed 15 repetitions obtaining a total of 120 repetitions manually feeding the machines with loads 
of around $100 \mathrm{~kg}$. In order to reduce the effect of the progressive blade wear, a random machine feeding sequence was used (Nati et al., 2010).

Productivity was determined measuring the exact time required to process the material excluding the machine-feed operation. The exact working time was calculated in the post-processing operation looking at the power adsorption graph.

In order to evaluate the weight of processed material a load cell between the metal bin used to collect the chips produced by the machine and its support was installed. In this way, for each repetition, was possible to know the exact weight of processed material and, in post-processing operation, to calculate the productivity.

All sensors were connected to a pc-based multichannel acquisition system that filter, elaborate, display and record the incoming signals. The instrument can acquire up to 8 analog channels and up to 24 digital channels with a maximum sample rate of $10 \mathrm{ks} \cdot \mathrm{s}^{-1}$. For this study the recording sampling rate was fixed to $200 \mathrm{~Hz}$ and were used 3 analog channel (torque meter and thermocouples) and 2 digital channels (speed sensor and fuel meter).

The acquisition software can be configured to make real-time computation on the basis of the acquired data. With these feature is possible directly evaluate other derived parameters as the instantaneous power delivered to the chipper and total fuel consumption. The acquisition system also permit graphical visualization of the acquired data for better monitoring and interpretation.

Figure 1 show a typical screen used during the tests. On the right a graphical representation of the instantaneous power (up) and fuel consumption (down), on the left, in numerical form, PT0 torque and rpm, engine power and rotational speed, fuel consumption. On the bottom the average values obtained during the test.

In the post-processing operation specific power and specific fuel consumption were computed using the equations reported below: specific power (SP) equation:

$$
S P=\frac{P w}{p}\left[k W h \cdot t^{-1}\right]
$$

where " $p$ " is the productivity in tons per hour and " $P w$ " is the average power required in $\mathrm{kW}$.

Specific fuel consumption (SFC) equation:

$$
S f c=\frac{c}{p}\left[d m^{3} \cdot t^{-1}\right]
$$

where " $c$ " is the fuel consumption in $\mathrm{dm}^{3} \cdot \mathrm{h}^{-1}$ and "p" is again the productivity in tons per hour.

All the recorded data were analyzed to extract the net values of power requirements and fuel consumption. To obtain the net value of these parameters were conducted some data acquisitions in idle conditions setting the tractor engine at the rated working conditions obtaining the results showed in Table 1.

Furthermore the exact processing time was determined on the basis of the power absorption level introducing a power threshold value that is equal to the average power needed when the chipper runs in idle conditions. Above this value we can assert that the machine is processing material and it is running status (Figure 2).

Threshold values are necessary to calculate the net values of power, fuel consumption, specific power an specific power consumption subtracting the threshold values to the gross data. Net values are useful to better compare results obtained with different machines that work in different conditions. With this approach is possible to evaluate the amount of power (or other quantity studied) that is due to the material processing operation, removing the "mask effect" introduced by the idle values.

\section{Results}

Table 2 reports the average values of net power adsorption of the two machines.

Table 1. Average values obtained in idle conditions.

\begin{tabular}{lccc} 
Device & Power $[\mathrm{kW}]$ & Fuel $\left[\mathrm{dm}^{3} \cdot \mathrm{h}^{-1}\right]$ & Speed $[\mathrm{rpm}]$ \\
Drum & 4.9 & 8.4 & 776.7 \\
Disc & 4.6 & 6.3 & 521.3 \\
\hline
\end{tabular}

Table 2. Average net power for disc and drum machines.

\begin{tabular}{lll} 
Average net power [kW] & Disc & Drum \\
Chestnut logs & 30.8 & 41.4 \\
Poplar branches & 17.0 & 23.9 \\
\hline Poplar logs & 33.3 & 41.1 \\
Poplar mill & 23.2 & 29.6 \\
\hline TOTAL & 26.1 & 34.0 \\
\hline
\end{tabular}

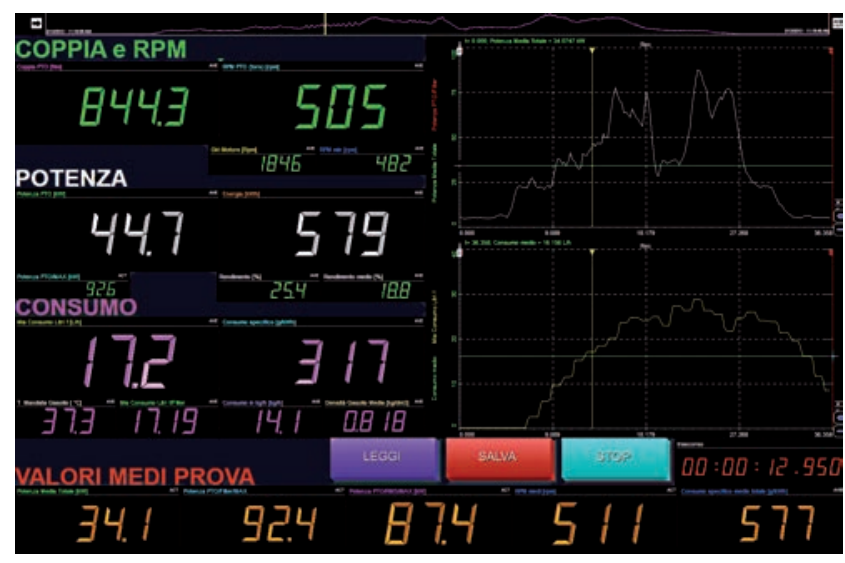

Figure 1. Typical acquisition system screen.

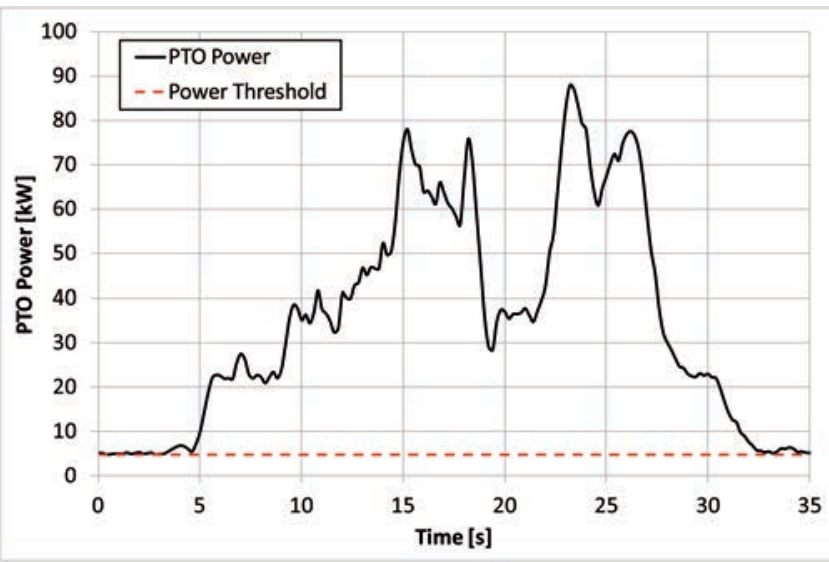

Figure 2. PTO power graph obtained processing a single $89 \mathrm{~kg}$ poplar logg. 
The drum tool machine requires the highest average net power (34.0 $\mathrm{kW}$ vs $26.1 \mathrm{~kW}$ obtained with disc tool) and the highest difference is in poplar branches processing were drum machine require up to $41 \%$ more power than disc machine. This suggest more difficulty to comminute this kind of material for drum machine.

Looking at the productivity (Table 3) poplar logs obtained higher values, but considering dry values, chestnut has the best values. This difference is due to the different levels of moisture content of the two materials.

Dry productivity was calculated considering the moisture content of each sample that were $44.7 \%$ for chestnut, $50 \%$ for poplar branches, $53 \%$ for poplar logs and $49 \%$ for poplar mill. Using dry productivity values is useful to compare the results with other machines removing the influence of the water content.

Table 4 shows the average values of specific fuel consumption obtained with the four different type of feedstock. The "fresh" column has been calculated considering the total weight of the material and the total fuel consumption. On the other hand, net dry values have been computed using the dry weight and net fuel consumption values (sub-

Table 3. Productivity values for disc and drum chippers.

\begin{tabular}{lcccc} 
Productivity $\left[\mathrm{t}^{-1} \mathbf{H}^{-1}\right]$ & \multicolumn{2}{c}{ Disc } & Dresh & Dry \\
\cline { 3 - 5 } Chestnut logs & Fresh & Dry & 12.3 & 6.7 \\
Poplar branches & 11.0 & 6.1 & 8.3 & 4.1 \\
\hline Poplar logs & 7.5 & 3.7 & 12.4 & 5.9 \\
Poplar mill & 11.7 & 5.5 & 8.0 & 4.2 \\
\hline TOTAL & 7.8 & 3.9 & 10.3 & 5.2 \\
\hline
\end{tabular}

Table 4. Average specific fuel consumption values.

\begin{tabular}{lcccc}
$\begin{array}{l}\text { Specific fuel } \\
\text { consumption } \\
{\left[\mathrm{dm}^{3} \cdot \mathrm{t}^{-1}\right]}\end{array}$ & Fresh & Net dry & Fresh & Net dry \\
Chestnut logs & 1.3 & 1,3 & 1.5 & 1,5 \\
Poplar branches & 1.5 & 1,1 & 1.7 & 1,3 \\
\hline Poplar logs & 1.3 & 1,5 & 1.5 & 1,8 \\
Poplar mill & 1.6 & 1,5 & 1.9 & 1,6 \\
\hline TOTAL & 1.4 & 1,3 & 1.7 & 1,5 \\
\hline
\end{tabular}

Table 5. Average specific energy.

\begin{tabular}{lcccc} 
Specific energy & \multicolumn{2}{c}{ Disc } & \multicolumn{2}{c}{ Drum } \\
{$\left[k_{W h} \mathbf{t}^{-1}\right]$} & Fresh & Net dry & Fresh & Net dry \\
Chestnut logs & 3.3 & 5,1 & 3.8 & 6,1 \\
Poplar branches & 3.0 & 4,6 & 3.5 & 5,8 \\
\hline Poplar logs & 3.3 & 6,1 & 3.7 & 7,0 \\
Poplar mill & 3.7 & 6,1 & 4.4 & 7,2 \\
\hline TOTAL & 3.3 & 5,5 & 3.8 & 6,5 \\
\hline
\end{tabular}

tracting the idle value). Poplar mill gives the higher "fresh" value for both machines and this may indicate a lower efficiency of the machines comminuiting this kind of feedstock. Looking at the "net dry" values drum machine has higher values $(+15 \%)$.

Another important efficiency indicator is the specific energy required to process the material. Table 5 reports average values of specific energy. Again, poplar mill requires the higher amount of specific energy, whereas poplar branches needs the lowest quantity of energy per unit of material processed.

Disc machine gives lower values of specific energy (average -15\%) with all kinds of feedstock-indicating a better efficiency in compared with the drum machine.

We have to focus that the values thus described are uniquely referred to the comminution work without delays and time needed by other operations like machine feeding. Delays may take up to $50 \%$ of the total working time (Spinelli and Visser, 2009) affecting heavily productivity and fuel consumption. This variation is a consequence of the operator effect that introduce a high variability in the measurement of this parameters as described by Harstela (1988).

In this study, to eliminate this effect, all the data were computed only during the comminution time interval not considering all the other accessory operation.

\section{Conclusions}

The average productivity obtained during the tests was $10.3 \mathrm{t} \cdot \mathrm{h}^{-1}$ for

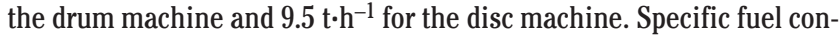
sumption was $1.7 \mathrm{dm}^{3} \cdot \mathrm{t}^{-1}$ and $1.4 \mathrm{dm}^{3} \cdot \mathrm{t}^{-1}$ for drum and disc tool respectively. These values can be compared with other chippers of similar size (Spinelli et al., 2012). Also the specific power adsorption values (average 3.3 and $3.8 \mathrm{kWh} \cdot \mathrm{t}^{-1}$ ) are aligned with those obtained with machines that adopt similar cutting system. These values, if compared with those obtained by Facello et al., (2012) testing a converted grinder, in general are lower and this suggest a different efficiency of the cutting technology used by the machine.

Using disc or drum machines give significant differences in terms of specific energy adsorption and productivity.

\section{References}

Chum, H.L., and Overend, R.P. (2001). Biomass and renewable fuels. Fuel Process. Technol. 71, 187-195.

Facello, A., Cavallo, E., and Spinelli, R. (2012). Chipping vs grinding, net energy requirements. In FORMEC 2012, (Cavtat), 8-12 september 2012.

Harstela, P. (1988). Principle of comparative time studies in mechanized forest work. Scand. J. For. Res. 3, 253-257.

Hillring, B. (2006). World trade in forest products and wood fuel. Biomass Bioene. 30, 815-825.

Nati, C., Spinelli, R., and Fabbri, P. (2010). Wood chips size distribution in relation to blade wear and screen use. Biomass Bioen. 34, $583-587$.

Spinelli, R., and Visser, R.J.M. (2009). Analyzing and estimating delays in wood chipping operations. Biomass Bioen. 33, 429-433.

Spinelli, R., Cavallo, E., and Facello, A. (2012). A new comminution device for high-quality chip production. Fuel Process. Technol. 99, $69-74$.

De Wit, M., and Faaij, A. (2010). European biomass resource potential and costs. Biomass Bioen. 34, 188-202. 